\title{
Successful clinical application of pre-implantation genetic diagnosis for infantile neuroaxonal dystrophy
}

\author{
YAN HAO $^{1-3 *}$, DAWEI CHEN ${ }^{1-3 *}$, GUIRONG ZHANG ${ }^{4 *}$, ZHIGUO ZHANG $^{1-3}$, XIAOJUN LIU $^{4}$, \\ PING ZHOU ${ }^{1-3}$, ZHAOLIAN WEI ${ }^{1-3}$, XIAOFENG XU ${ }^{1-3}$, XIAOJIN HE$^{1-3}$, LIXIAN XING ${ }^{4}$, MINGRONG LV $^{1-3}$, \\ DONGMEI JI ${ }^{1-3}$, BEILI CHEN ${ }^{1-3}$, WEIWEI ZOU ${ }^{1-3}$, HUAN WU ${ }^{1-3}$, YAJING LIU ${ }^{1-3}$ and YUNXIA CAO ${ }^{1-3}$ \\ ${ }^{1}$ Department of Obstetrics and Gynecology, Reproductive Medicine Center, The First Affiliated Hospital of Anhui \\ Medical University; ${ }^{2}$ Anhui Province Key Laboratory of Reproductive Health and Genetics, Anhui Medical University, \\ Hefei, Anhui 230032; ${ }^{3}$ Department of Biopreservation, Anhui Provincial Engineering Technology Research Center \\ for Biopreservation and Artificial Organs, Hefei, Anhui 230027; ${ }^{4}$ Department of Genetics, \\ Peking Medriv Academy of Genetics and Reproduction, Beijing 102629, P.R. China
}

Received September 14, 2017; Accepted July 30, 2019

DOI: $10.3892 /$ etm.2019.8302

\begin{abstract}
Infantile neuroaxonal dystrophy (INAD) is a rare, lethal, autosomal recessive neurodegenerative disease and leads to progressive impairment of movement and cognition. A couple with a proband child with calcium-independent group VI phospholipase A2 (PLA2G6)-associated INAD and a previous affected pregnancy sought pre-implantation genetic diagnosis (PGD) to bear a healthy child. Intracytoplasmic sperm injection treatment was performed and 15 blastocystic embryos were obtained at days 5 and 6, and these biopsies were amplified. PGD was performed by next-generation sequencing-based linkage analysis in conjunction with aneuploidy screening. Only two embryos were considered for transfer. In the second frozen-thawed embryo transfer cycle, transfer of a mosaic PLA2G6 c.692G>T heterozygous embryo resulted in a singleton ongoing pregnancy. Prenatal diagnosis was performed using amniotic fluid cells, providing results consistent with those of PGD. The aneuploidy screen and karyotype analysis indicated that the chromosomes of the fetus were normal without any mosaicism. The present study reported the first successful PGD for INAD. For parents at risk, this strategy may successfully lead to pregnancies with embryos unlikely to develop INAD, thus providing valuable experience in reproductive management regarding INAD and potentially other single-gene disorders.
\end{abstract}

Correspondence to: Professor Yunxia Cao, Department of Obstetrics and Gynecology, Reproductive Medicine Center, The First Affiliated Hospital of Anhui Medical University, 218 Jixi Road, Hefei, Anhui 230032, P.R. China

E-mail: caoyunxia6@126.com

${ }^{*}$ Contributed equally

Key words: next-generation sequencing, infantile neuroaxonal dystrophy, pre-implantation genetic diagnosis, linkage analysis, single nucleotide polymorphism, aneuploidy

\section{Introduction}

Infantile neuroaxonal dystrophy (INAD; Online Mendelian Inheritance in Man no. 256600), is an extremely rare autosomal recessive neurodegenerative disorder involving axons in the central and peripheral nervous system. The diagnosis of INAD is difficult due to the frequent occurrence of atypical cases and lack of specific early signs (1). The clinical manifestation includes progressive psychomotor regression with onset between 6 months and 2 years of age, and usually leads to death by the age of 10 years (2). Most patients with INAD display a progressive disorder with motor and mental deterioration, cerebellar ataxia, spastic tetraplegia, hyperreflexia and early visual disturbances (3).

INAD is caused by loss of the ability of calcium-independent group VI phospholipase A2 (PLA2G6) to catalyze fatty acid release from phospholipids (4). The PLA2G6 gene is mapped to chromosome $22 \mathrm{q}$ and encodes a $85-\mathrm{kDa}$ protein, which is also known as calcium-independent phospholipase A2 $\beta$ (5). At present, no effective treatments are available for INAD. The carrier couple had a child affected by INAD, which implies a $25 \%$ recurrence risk for future pregnancies. Preimplantation genetic diagnosis (PGD) is a powerful tool for preventing this neurodegenerative disorder without facing the trauma of termination of pregnancy in the case of an affected fetus.

PGD as an alternative to current prenatal diagnoses for severe lethal inherited diseases has been in use for $>2$ decades since its introduction by Handyside et al (6) in 1990. The technique has been used not only for single-gene disorders (SGD) to avoid the risk of having an affected child (7), but also for chromosomal abnormalities, human leukocyte antigen matching (8), mitochondrial disease (9) and hereditary cancer syndrome (10).

Compared to prenatal diagnosis, PGD spares parents from the physical and emotional trauma of the termination of pregnancy in the case of an affected fetus. The sensitivity and efficiency of PGD to detect SGDs is restricted by amplification 
failure or allelic dropout due to the limited amount of template DNA in a single embryonic cell (11). To solve these problems, blastocyst biopsy for inputting more cells and whole genome amplification (WGA) combined with linkage analysis (haplotyping analysis) have been applied to improve the accuracy of PGD $(12,13)$.

Linkage analysis is a method to deduce the inheritance of mutation alleles by detecting short tandem repeats (STRs) or single nucleotide polymorphisms (SNPs). The STR approach is not only labor-intensive and time-consuming, but also often less informative due to the limited number and uneven distribution of polymorphic markers in certain pedigrees (14). The introduction of karyomapping (15-17) hold promise to markedly change the way molecular diagnostics are performed during PGD with higher efficiency, accuracy and reliability. Karyomapping technology for PGD makes use of the abundance of the 'informative' SNPs in pedigrees, which allows for simultaneous detection of monogenic and chromosomal disorders. However, karyomapping has its limitations. For instance, for certain genes, which have low SNP coverage, polymerase chain reaction (PCR) testing, including STR analysis or direct mutation detection, is required to be performed in parallel (18). Thus, it would be more practical if specific SNPs were to be identified for rare inherited diseases with higher SNP densities near the causal genes (14).

With the advent of next-generation sequencing (NGS) techniques, an opportunity arises to perform cost-efficient genetic testing by sequencing in different clinical scenarios, which may contribute to definitive improvements in the genetic assessment of embryos prior to transfer to the uterus (19). NGS-based methods, including Mutated Allele Revealed by Sequencing with Aneuploidy and Linkage Analyses and haplotyping analysis, have been successfully applied in SGDs and chromosomal copy number assessment $(7,18,20,21)$.

In the present study, NGS-based haplotyping analysis combined with aneuploidy screening was applied in the PGD for INAD. The patient got pregnant successfully in the second frozen-thawed embryo transfer cycle. To the best of our knowledge, the present study is the first to report on PGD for INAD.

\section{Materials and methods}

Participants. The couple (maternal age, 26 years and paternal age, 27 years) was referred to the Reproductive Medicine Center of The First Affiliated Hospital of Anhui Medical University (Hefei, China) due to an adverse birth history in two cases (Fig. 1). Their first son suffered from dystonia at 2 years of age, followed by the development of retardation, and he died at 5 years of age with a diagnosis of INAD. DNA testing of the son by targeted-next generation sequencing of PLA2G6 revealed the compound heterozygous mutations c.692G $>\mathrm{T}$ (p.G231V) in exon 5 and c.2213_2220delCAGACGGG (p.Asp739GlyfsX29) in exon 16. The parents, with their son, had visited the Department of Neurology in Beijing Children's Hospital (Beijing, China) in February 2012 and the gene diagnosis of their first son had been confirmed there. Segregation analysis revealed the c.692G $>\mathrm{T}$ mutation in exon 5 in the mother and the c.2213_2220delCAGACGGG mutation in exon 16 in the father in a heterozygous state. The genetic

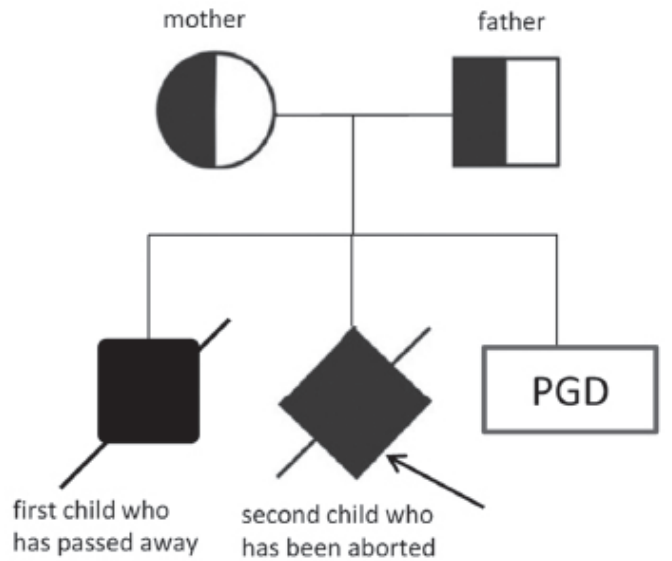

Figure 1. Pedigree of the family of the present study. PGD, pre-implantation genetic diagnosis. Arrow, second aborted child.

diagnosis of amniocentesis for prenatal diagnosis during the second pregnancy in May 2014 revealed the same compound heterozygous mutations as those in their first son and labor induction was therefore performed. The couple received counselling regarding PGD in Reproductive Medicine Center of the First Affiliated Hospital of Anhui Medical University in March 2016 in order to have a child without INAD. This study underwent an EMRO process

Fertilization, embryo culture, biopsy, vitrification and WGA. A long protocol was used for ovarian stimulation. Following ovarian stimulation, follicles were aspirated at 34-36 h after human chorionic gonadotropin injection and fertilized by intracytoplasmic sperm injection (ICSI). The following morning (day 1), each injected oocyte was checked for pronuclei to confirm fertilization. All embryos were cultured to the blastocyst stage (day 5 or 6) and scored according to Gardner's grading scale (22). Hatched blastocysts were biopsied using a $30-\mu \mathrm{m}$ inner diameter biopsy pipette (Cook Medical, Bloomington, IN, USA) with the ZILOS-tk laser (Hamilton Thorne, Inc., Beverly, MA, USA). Biopsied trophectoderm cells were then transferred to sterile $0.2-\mathrm{ml}$ PCR tubes supplemented with $2.5 \mu \mathrm{l} \mathrm{PBS}$, which was later subjected to whole-genome amplification (WGA). Biopsied blastocysts were then vitrified according to the protocol recommended in the Kitazato vitrification kit using Kitazato vitrification solution (Kitazato Biopharma Co. Ltd., Shizuoka, Japan). Multiple displacement amplification (MDA) was performed using a REPLI-g Single Cell kit (Qiagen, Hilden, Germany) for whole-genome amplification.

Linkage analysis by NGS-based SNP haplotyping and aneuploidy screening. Regarding the PLA2G6 gene (GenBank ID, NM_003560.2; chr22:38507502-38577857; reverse transcription product length, $70 \mathrm{~Kb}$ ) as a target region, a panel of 100 high-frequency SNP markers located $2 \mathrm{Mb}$ upstream and downstream of the PLA2G6 gene in the genomes of Han Chinese in Beijing and Southern Han Chinese from the 1,000 Genomes Project and the maternal mutation site were selected for NGS-based SNP haplotyping. Primers were designed using AmpliSeq Designer (https://www.ampliseq. com). Target regions were amplified by multiplex PCR (23). 


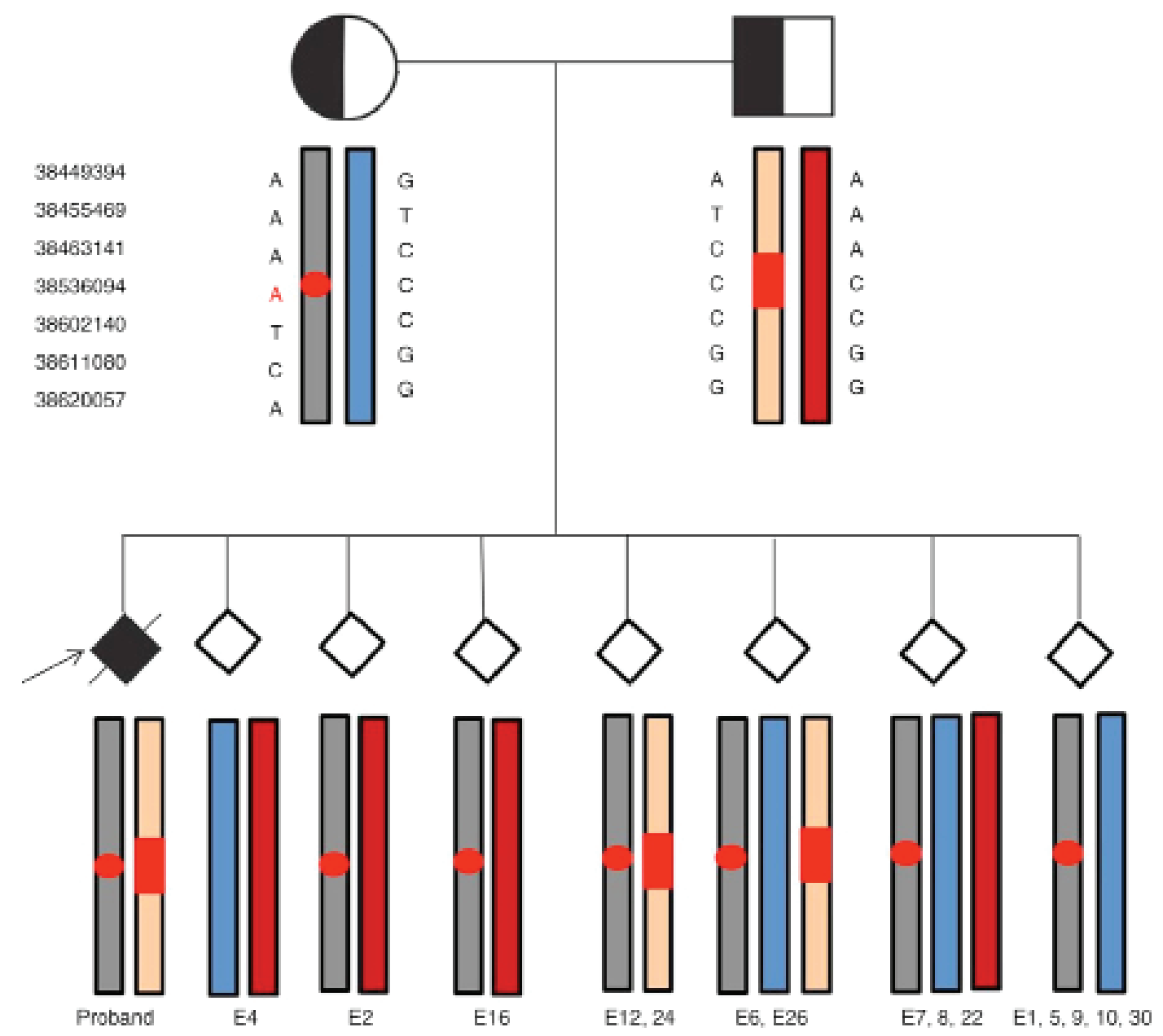

Figure 2. Next-generation sequencing-based single-nucleotide polymorphism haplotyping for infantile neuroaxonal dystrophy diagnosis. Gray and blue represent pathogenic and normal haplotypes of the mother, respectively, while beige and red represent the pathogenic and normal haplotypes of the father, respectively. Gene mutation sites were marked with red circles (maternal) and red rectangles (paternal). E4 was genotypically normal, embryos 2 and 16 exhibited a carrier pattern, and E12 and -24 were affected. E6, -26, -7, -8 and -22 were diagnosed as having trisomies. Uniparental disomy occurred in E1, -5, -9, -10 and -30. E, embryo.

The WGA products and designed primers were used for library preparation with Ion AmpliSeq Library Kits (Thermo Fisher Scientific, Inc., Waltham, MA, USA). The template preparation was performed using an Ion One Touch 2 system and an Ion One Touch ES following instructions of the latest version of the manuals (Ion Onetouch Template kit; Thermo Fisher Scientific, Inc.). The template positive Ion Sphere Particles were sequenced on an Ion Torrent Personal Genome Machine (PGM) (Life Technologies Ltd.) according to the instructions of the Ion Sequencing kit v2.0 (Thermo Fisher Scientific, Inc.). The remaining WGA products were simultaneously subjected to aneuploidy screening by NGS according to a standard protocol using the Ion Torrent System (Thermo Fisher Scientific, Inc.) as previously described (24). The data from the PGM sequencing were analyzed by Peking Jabrehoo Med Tech., Ltd. (Beijing, China). Sanger sequencing for the paternal mutation sites was performed in order to verify the NGS results.

Blastocysts thawing and transfer. At three months after oocyte retrieval, the patient was treated with oral Estradiol Valerate from day 3 to prepare the endometrium for frozen embryo transfer. Luteal support by administration of intramuscular progesterone was applied when a satisfactory endometrial development (thickness, $\geq 8 \mathrm{~mm}$ ) was confirmed on ultrasound. The embryo unaffected by INAD, which had normal chromosomes or mosaicism, was thawed and transferred. Clinical pregnancy was confirmed when an intrauterine gestational sac with heartbeat was observed by ultrasound examination at 35 and 65 days after embryo transfer. Amniocentesis was performed at 18 weeks of gestation. Linkage analysis and aneuploidy screen were performed using NGS. Karyotype analysis was also performed.

\section{Results}

ICSI, biopsy and MDA. A total of 40 cumulus-oocyte complexes were retrieved, and 40 metaphase-II oocytes were subjected to ICSI for fertilization. A total of 15 embryos reached the hatched blastocyst stage of development on day 5 or 6 post-ICSI. Biopsies (5-10 trophoblastic ectoderm cells) from all blastocysts were successfully amplified by MDA.

Linkage analysis by NGS-based SNP haplotyping and $N G S$-based aneuploidy screen. Based on the SNPs in the proband, heterozygote SNPs in the father and heterozygote 


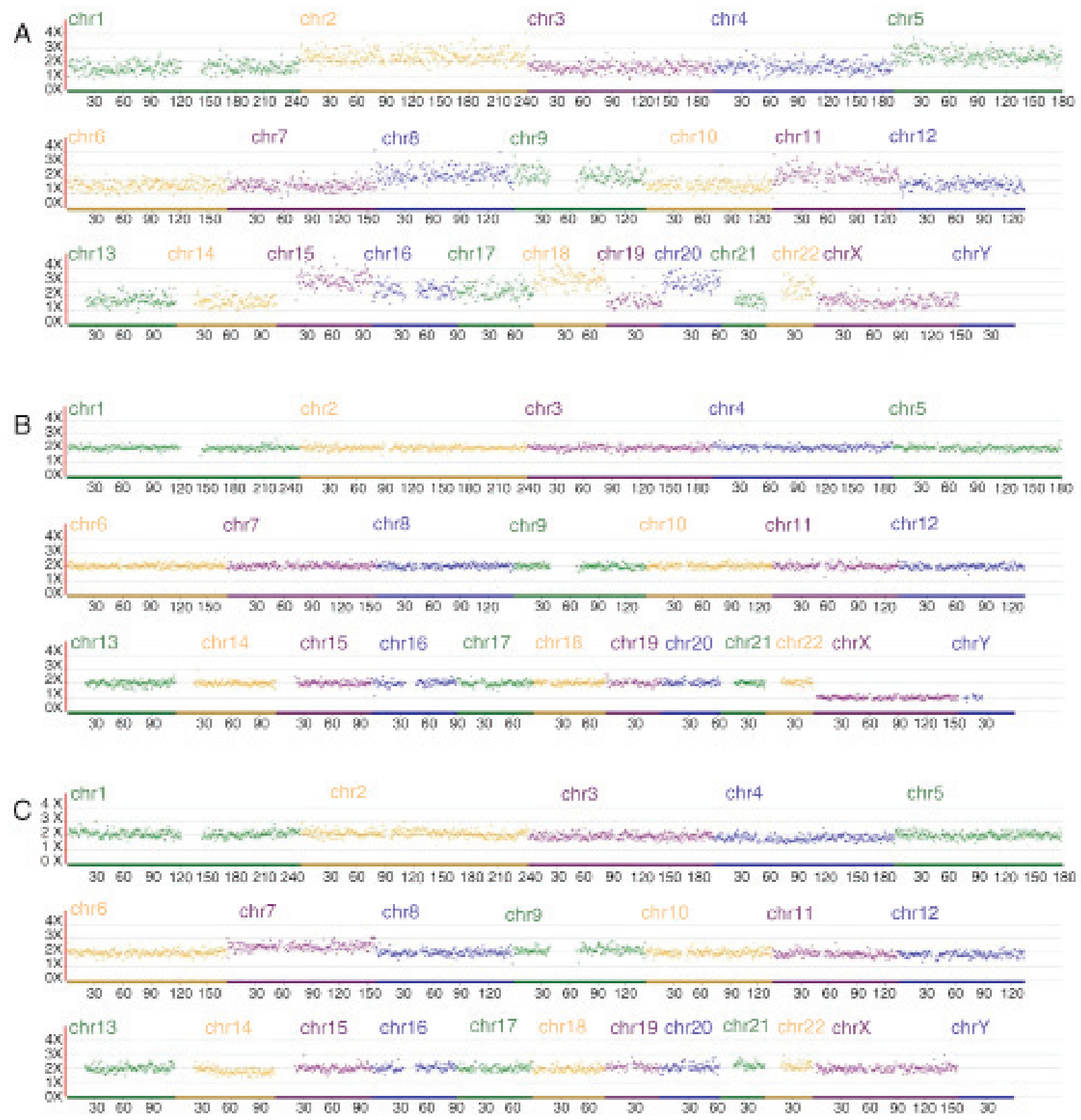

Figure 3. NGS-based aneuploidy screen for embryos. (A) NGS-based aneuploidy screen for embryo 1: Segmental imbalances for chr 2, 5, 8, 9, 11, 15, 16, 18, 20 and 22. (B) NGS-based aneuploidy screen for embryo 4: Normal. (C) NGS-based aneuploidy screen for embryo 2: Mosaicism for trisomy 7. NGS, next-generation sequencing; chr, chromosome.

SNPs in the mother were selected at the same loci to construct the haplotypes of the blastocysts. The haplotypes were constructed by including 77 informative SNPs from 100 selected high-frequency SNP markers and the maternal mutation site (Fig. 2). The remaining MDA products were simultaneously subjected to aneuploidy screening (Fig. 3; Table I).

Embryo 4 was diagnosed as being unaffected by INAS and exhibiting euploidy. Embryos 2 and 16 were diagnosed as carriers of INAS, with embryo 2 inheriting the maternal affected haplotype and the normal paternal haplotype, whereas embryo 16 inheriting the paternal affected haplotype and the normal maternal haplotype. Embryos 12 and 24 had pathogenic haplotypes, inheriting both affected parental haplotypes similar to the previous affected offspring. Embryos 6, 7, 8, 22 and 26 were diagnosed as having trisomy. Embryos 1, 5, 9, 10 and 30 were identified to have uniparental disomy (UPD), inheriting both maternal haplotypes. NGS-based aneuploidy screening revealed that embryos $4,5,7,9,10,12$ and 24 were normal and embryo 2 displayed mosaicism for trisomy 7 (Fig. 3). Sanger sequencing analysis of the paternal mutation site indicated that embryos 2 and 4 were void of this mutation site (Figs. 4 and 5). On the basis of the linkage analysis and aneuploidy results, only embryos 2 and 4 were considered for transfer.

Clinical outcome. The unaffected embryo 4 was successfully thawed three months after it was generated. However, 


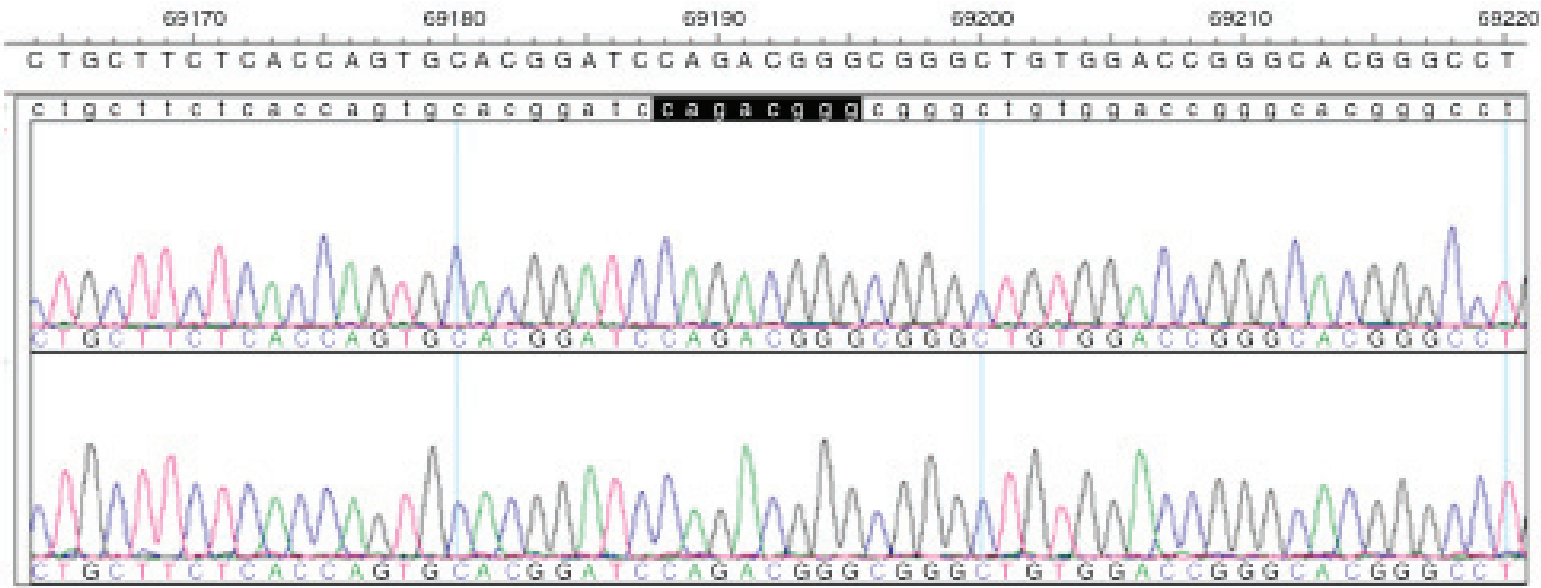

Figure 4. Sanger sequencing of paternal mutation site on embryo 4: Absence of mutation site (c.2213_2220delCAGACGGG).

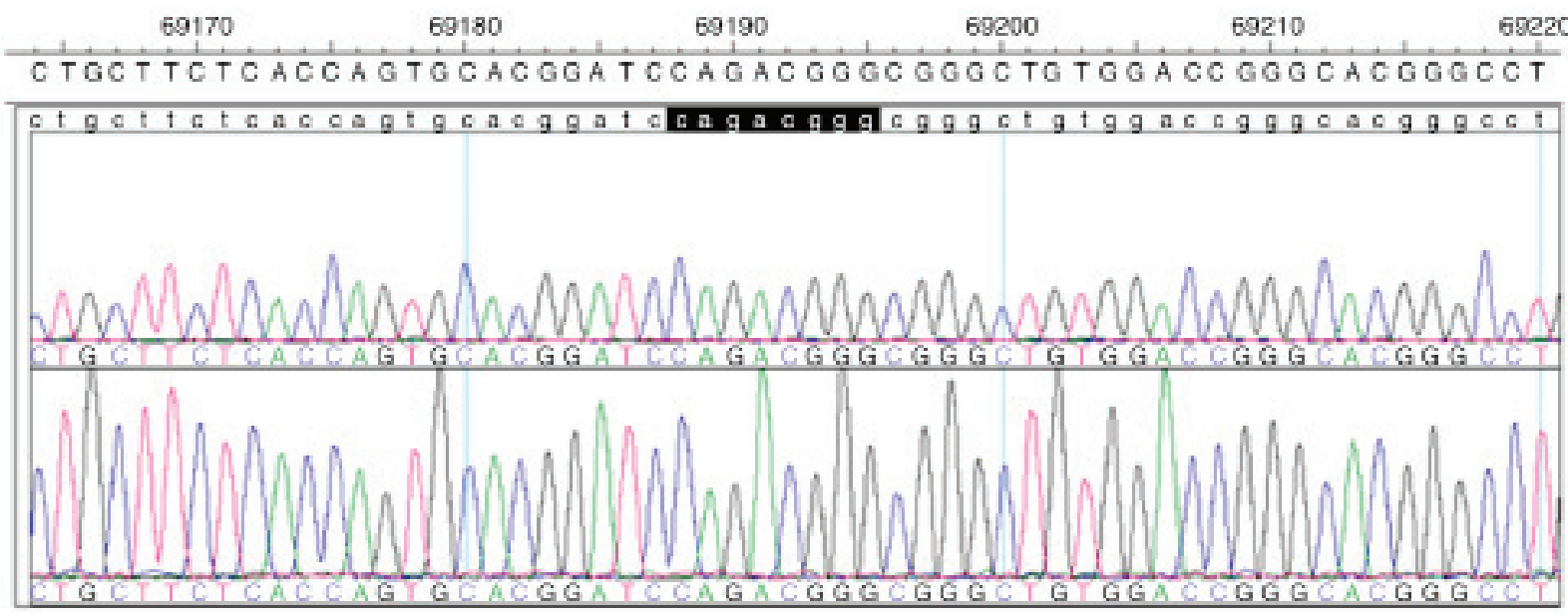

Figure 5. Sanger sequencing of paternal mutation site on embryo 5: Absence of mutation site (c.2213_2220delCAGACGGG).

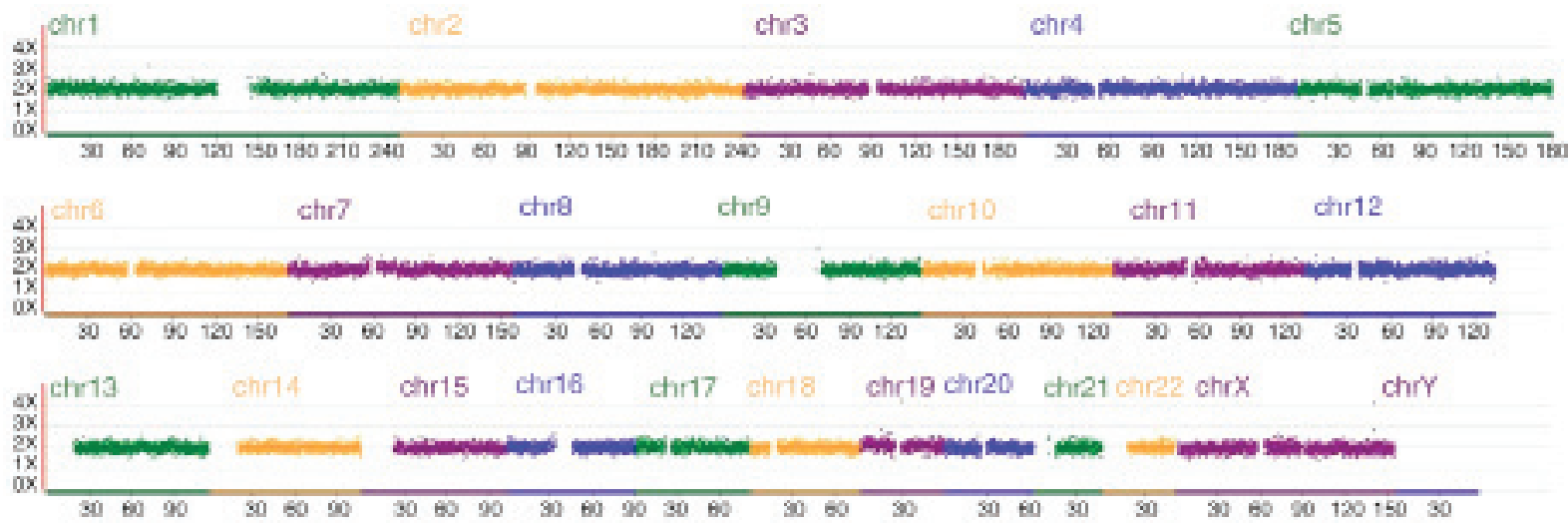

Figure 6. Result of aneuploidy screen (transfer of embryo 2) of the amniotic fluid cells: Normal. Chr, chromosome.

no clinical pregnancy was achieved. In the subsequent frozen-thawed cycle, the couple asked for transfer of the carrier embryo 2, although it was mosaic. The couple informed of relevant risks prior to transfer and a consent form was signed. Embryo 2 was then transferred and resulted in a successful pregnancy. Transvaginal ultrasonography examination on day 35 and 65 revealed a single intrauterine gestational sac with a normal fetal heartbeat. Prenatal diagnosis was performed using amniotic fluid cells, whose results were consistent with those of the PGD. The aneuploidy screen (Fig. 6) and karyotype analysis indicated that the chromosomes of the fetus were normal and not mosaic. The full-term female baby was born by normal Apgar score, with a birth weight of $3600 \mathrm{~g}$, and a head circumference was $50 \mathrm{~cm}$. 
Table I. Summary of pre-implantation genetic diagnosis results regarding infantile neuroaxonal dystrophy.

\begin{tabular}{|c|c|c|}
\hline Embryo no. & NGS-based linkage analysis & NGS-based aneuploidy screening \\
\hline 1 & UPD & $\begin{array}{l}+2(\mathrm{p} 25.3->\mathrm{p} 25.1)(9.82 \mathrm{Mb}) \\
+5(\mathrm{p} 15.33->\mathrm{q} 13.3)(71.48 \mathrm{Mb}) \\
+8(\mathrm{q} 22.3->\mathrm{q} 24.3)(33.71 \mathrm{Mb}) \\
+9(\mathrm{p} 24.3->\mathrm{p} 21.1)(29.72 \mathrm{Mb}) \\
+11(\mathrm{p} 15.1->\mathrm{p} 14.1)(8.71 \mathrm{Mb}) \\
+15(\mathrm{q} 11.1->\mathrm{q} 26.3)(78.30 \mathrm{Mb}) \\
+16(\mathrm{p} 13.3->\mathrm{p} 11.2)(23.34 \mathrm{Mb}) \\
+18(\mathrm{p} 11.32->\mathrm{q} 23)(73.91 \mathrm{Mb}) \\
+20(\mathrm{p} 13->\mathrm{q} 13.33)(59.93 \mathrm{Mb}) \\
+22(\mathrm{q} 11.1->\mathrm{q} 13.33)(33.13 \mathrm{Mb})\end{array}$ \\
\hline 2 & Carrier & Mosaicism for trisomy 7 (q33->q36.3) $(21.50 \mathrm{Mb})$ \\
\hline 4 & Normal & Normal \\
\hline 5 & UPD & Normal \\
\hline 6 & Trisomy & $\begin{array}{l}+4(\mathrm{p} 15.1->\mathrm{q} 34.3)(136.59 \mathrm{Mb}) \\
+6(\mathrm{p} 25.3->\mathrm{p} 21.1)(42.11 \mathrm{Mb}) \\
+8(\mathrm{p} 23.3->\mathrm{q} 21.11)(72.54 \mathrm{Mb}) \\
+12(\mathrm{q} 24.11->\mathrm{q} 24.31)(14.08 \mathrm{Mb})\end{array}$ \\
\hline 7 & Trisomy & Normal \\
\hline 8 & Trisomy & $\begin{array}{l}+2(\mathrm{q} 37.1->\mathrm{q} 37.3)(7.14 \mathrm{Mb}), \\
+3(\mathrm{p} 26.3->\mathrm{p} 22.2)(36.54 \mathrm{Mb}), \\
+3(\mathrm{p} 13->\mathrm{q} 29)(120.18 \mathrm{Mb}) \\
-X(\mathrm{q} 11.1->\mathrm{q} 28)(88.10 \mathrm{Mb})\end{array}$ \\
\hline 9 & UPD & Normal \\
\hline 10 & UPD & Normal \\
\hline 12 & Pathogenic & Normal \\
\hline 16 & Carrier & $\begin{array}{l}+1(\mathrm{p} 36.13->\mathrm{p} 35.2)(11.94 \mathrm{Mb}) \\
+2(\mathrm{p} 23.1->\mathrm{p} 22.3)(4.21 \mathrm{Mb}) \\
-4(\mathrm{q} 33->\mathrm{q} 34.3)(7.25 \mathrm{Mb}) \\
+5(\mathrm{p} 13.3->\mathrm{q} 14.3)(56.95 \mathrm{Mb}) \\
+6(\mathrm{p} 22.3->\mathrm{p} 21.31)(12.90 \mathrm{Mb})\end{array}$ \\
\hline 22 & Trisomy & Abnormal \\
\hline 24 & Pathogenic & Normal \\
\hline 26 & Trisomy & $\begin{array}{l}+1(\mathrm{p} 36.21->\mathrm{p} 32.1)(45.95 \mathrm{Mb}) \\
-2(\mathrm{p} 25.1->\mathrm{p} 24.2)(6.64 \mathrm{Mb}) \\
+3(\mathrm{p} 26.3->\mathrm{p} 25.2)(11.43 \mathrm{Mb}) \\
-4(\mathrm{q} 12->\mathrm{q} 13.1)(5.49 \mathrm{Mb})\end{array}$ \\
\hline 30 & UPD & $\begin{array}{l}-1(\mathrm{p} 36.21->\mathrm{p} 36.12)(5.66 \mathrm{Mb}) \\
+1(\mathrm{q} 25.1->\mathrm{q} 25.2)(5.58 \mathrm{Mb}) \\
+2(\mathrm{p} 24.1->\mathrm{p} 23.2)(6.26 \mathrm{Mb}) \\
-3(\mathrm{p} 26.3->\mathrm{p} 25.1)(15.52 \mathrm{Mb}) \\
+4(\mathrm{q} 21.1->\mathrm{q} 22.1)(13.23 \mathrm{Mb})\end{array}$ \\
\hline
\end{tabular}

NGS, next-generation sequencing; UPD, uniparental disomy.

\section{Discussion}

To the best of our knowledge, the present study was the first to report on PGD in parallel with an aneuploidy screen for INAD.

INAD is an extremely rare, lethal, autosomal recessive neurodegenerative disorder. Disease progression is rapid, and numerous affected children never learn to walk or lose the ability shortly after attaining it. Severe spasticity, progressive cognitive decline and visual impairment typically result in death during the first decade of life and no effective treatments are available, thus emphasizing the importance of preventing this lethal disease (25). In the present study, during PGD, detection of SNPs in the vicinity of the mutation by NGS offers the advantage of a one platform technology with 
less processing of samples and lower labor costs. Compared with previous methods of linkage analysis by short tandem repeats (STR), NGS-based SNP haplotyping provides more informative genetic markers in high throughput (7) and the inclusion of more multiple linked SNPs close to the targeted mutation in NGS allows for more accurate measurement than STR. On the basis of NGS-based linkage analysis, trisomies and monosomies may be identified by the presence of both haplotypes from one parent or absence of either chromosome haplotype from the parent of origin (26). In the present study, NGS-based linkage analysis revealed trisomies in five embryos (nos. 6, 7, 8, 22 and 26), with the extra haplotype being the maternal one.

Furthermore, when compared to other techniques, including array-based comparative genomic hybridization (CGH) and quantitative fluorescent PCR, this method has the advantage that general features of the proband's haplotype may be detected, including UPD and recombination. For embryos $1,5,9,10$ and 30 , examination of the NGS revealed that no paternal chromosomes were present and both haplotypes were from the maternal pair, which is called heterodisomy. UPD for a complete chromosome may appear due to post-fertilization error, gamete complementation, trisomic/monosomic rescue, mitotic error, isochromosome formation, deletion and duplication (27). Segmental UPD may arise from a postzygotic somatic recombination between the maternal and paternal homologue, or in connection with numerical and/or structural chromosomal aberrations (27). It is also a novel mechanism for the occurrence of INAD (28). In the present study, 5 embryos were diagnosed with UPD by NGS-based SNP haplotyping, exemplifying the high efficiency of this technique.

Given the high prevalence of embryonic aneuploidy, particularly in mothers of advanced reproductive age, unaffected embryos remain at high risk of implantation failure or pregnancy loss due to aneuploidy (29). Therefore, single-gene PGD in conjunction with aneuploidy screening is recommended, as a normal genotyping result does not necessarily guarantee that the embryo is also euploid. The importance of screening for aneuploidy and SGDs were also demonstrated in the present study. Embryos 1, 6, 8, 16, 22, 26 and 30 were aneuploidy. As sequencing costs decrease further, allowing for a greater read depth per sample for the same or a reduced price, NGS approaches provide simultaneous evaluation of single-gene disorders and translocations with comprehensive aneuploidy screening from the same biopsy without the requirement for multiple technological platforms $(7,18,30,31)$. Compared to array CGH or SNP array, the NGS approach not only detects mosaic blastocysts, but may also be effective in characterizing small abnormal chromosomal fragments (24). In the present study, NGS-based 24-aneuploidy screening revealed that embryo 2 was mosaic; the method allows for accurate detection of segmental imbalances as small as $\sim 4$ $\mathrm{Mb}$ in size due to the high resolution. During the second frozen-thawed embryo transfer cycle, although embryo 2 was mosaic for trisomy 7 , it had the potential to achieve a full-term pregnancy (32-34). The couple had a strong demand for transferring this mosaic embryo and were informed of relevant risks. Fortunately, the result of the prenatal diagnosis revealed that the chromosomes were normal, indicating the value of this mosaic embryo.
Nowadays, karyomapping has the potential for providing a simultaneous identification of aneuploidy and SGD, but since karyomapping has not yet been fully validated for this purpose, it was decided that array CGH is used in parallel in the present study, in order to provide information regarding the chromosomal status (15). Karyomapping is not validated for microdeletions (26), and cannot detect sequence-identical chromosome duplication that may result from malsegregation of chromosomes during the early cleavage divisions of the embryo (1). Furthermore, the dependence of DNA samples from family members may limit its application. NGS-based linkage analysis may still correctly diagnose the embryos by using the affected embryo as the proband under the circumstance of the absence of suitable affected family members (18). Chromosomal copy number assessment based on NGS may offer two major advantages: i) Enhanced detection of partial or segmental aneuploidies as a result of the potential increase in chromosomal analysis resolution to a few $\mathrm{Mb}$, and ii) the potential automation of the sequencing library preparation to minimize human errors, reduce hands-on time, and achieve higher throughput and consistency $(35,36)$.

In summary, the present study was the first to report on successful PGD for INAD. The feasibility of an NGS-based linkage analysis method for the selection of embryos for couples carrying PLA2G6 mutations was demonstrated. With reduced cost, it is expected that NGS-based analysis may enable PGD in parallel with aneuploidy screen for all types of monogenetic disorders with a known pathogenic gene mutation, thus preventing the occurrence of severe genetic diseases, which will ultimately bring benefits for the whole population.

\section{Acknowledgements}

Not applicable.

\section{Funding}

The current study is financially supported through grants from Anhui provincial science and technology research project (grant no. 1604a0802077), College Natural Science Project of Anhui Province (grant no. KJ2019A0287) and the Central Guiding Science and Technology Development of the Local Anhui Province (grant no. 2018080802D0081).

\section{Availability of data and materials}

All data generated or analyzed during the present study are included in this published article.

\section{Authors' contributions}

YH contributed to writing the manuscript, preparation of the manuscript and whole-genome amplification. DC, GZ, XL and LX contributed to NGS data analysis. ZZ contributed to embryo biopsy and embryo culture. PZ, ZW, XX and YC collected the data of the patient, performed ovarian stimulation and revised the manuscript. XH, ML, DJ, BC performed NGS. WZ, HW and YL contributed to the literature review and vitrified embryos. All authors read and approved the final manuscript. 


\section{Ethical approval and consent to participate}

The study was approved by the Ethics Committees of the Anhui Medical University (approval no. 2017002). This study underwent an EMRO process. The patients provided written informed consent for undertaking PGD. Furthermore, the parents provided written informed consent regarding the use of any embryos that are not viable for implantation for scientific research.

\section{Patient consent for publication}

Not applicable.

\section{Competing interests}

The authors declare that they have no competing interests.

\section{References}

1. Iodice A, Spagnoli C, Salerno GG, Frattini D, Bertani G, Bergonzini P, Pisani F and Fusco C: Infantile neuroaxonal dystrophy and PLA2G6-associated neurodegeneration: An update for the diagnosis. Brain Dev 39: 93-100, 2017.

2. Wu Y, Jiang Y, Gao Z, Wang J, Yuan Y, Xiong H, Chang X, Bao X, Zhang Y, Xiao J and Wu X: Clinical study and PLA2G6 mutation screening analysis in Chinese patients with infantile neuroaxonal dystrophy. Eur J Neurol 16: 240-245, 2009.

3. Khateeb S, Flusser H, Ofir R, Shelef I, Narkis G, Vardi G, Shorer Z, Levy R, Galil A, Elbedour K and Birk OS. PLA2G6 mutation underlies infantile neuroaxonal dystrophy. Am J Hum Genet 79: 942-948, 2006.

4. Engel LA, Jing Z, O'Brien DE, Sun M and Kotzbauer PT: Catalytic function of PLA2G6 is impaired by mutations associated with infantile neuroaxonal dystrophy but not dystonia-parkinsonism. PLoS One 5: e12897, 2010.

5. Morgan NV, Westaway SK, Morton JE, Gregory A, Gissen P, Sonek S, Cangul H, Coryell J, Canham N, Nardocci N, et al: PLA2G6, encoding a phospholipase A2, is mutated in neurodegenerative disorders with high brain iron. Nat Genet 38: 752-754, 2006.

6. Handyside AH, Kontogianni EH, Hardy K and Winston RM: Pregnancies from biopsied human preimplantation embryos sexed by Y-specific DNA amplification. Nature 344: 768-770, 1990.

7. Treff NR, Fedick A, Tao X, Devkota B, Taylor D and Scott RT Jr: Evaluation of targeted next-generation sequencing-based preimplantation genetic diagnosis of monogenic disease. Fertil Steril 99: 1377-1384.e6, 2013.

8. Kurekci E, Küpesiz A, Anak S, Öztürk G, Gürsel O, Aksoylar S, Ileri T, Kuşkonmaz B, Eker İ, Cetin M, et al: Hematopoietic stem cell transplantation using preimplantation genetic diagnosis and human leukocyte antigen typing for human leukocyte antigen-matched sibling donor: A Turkish Multicenter Study. Biol Blood Marrow Transplant 23: 790-794, 2017.

9. Sallevelt SC, Dreesen JC, Drüsedau M, Hellebrekers DM, Paulussen AD, Coonen E, van Golde RJ, Geraedts JP, Gianaroli L, Magli MC, et al: PGD for the m.14487 T>C mitoch ondrial DNA mutation resulted in the birth of a healthy boy. Hum Reprod 32: 698-703, 2017.

10. Lee VC, Chow JF, Lau EY, Kwong A, Leung SY, Yeung WS $\mathrm{Ho} \mathrm{PC}$ and $\mathrm{Ng}$ EH: Preimplantation genetic diagnosis for hereditary cancer syndrome: Local experience. Hong Kong Med J 22: 289-291, 2016.

11. Dreesen J, Destouni A, Kourlaba G, Degn B, Mette WC, Carvalho F, Moutou C, Sengupta S, Dhanjal S, Renwick P, et al: Evaluation of PCR-based preimplantation genetic diagnosis applied to monogenic diseases: A collaborative ESHRE PGD consortium study. Eur J Hum Genet 22: 1012-1018, 2014.

12. Shen J, Cram DS, Wu W, Cai L, Yang X, Sun X, Cui Y and Liu J: Successful PGD for late infantile neuronal ceroid lipofuscinosis achieved by combined chromosome and TPP1 gene analysis. Reprod Biomed Online 27: 176-183, 2013.
13. Trachoo O, Satirapod C, Panthan B, Sukprasert M, Charoenyingwattana A, Chantratita W, Choktanasiri W and Hongeng S: First successful trial of preimplantation genetic diagnosis for pantothenate kinase-associated neurodegeneration. J Assist Reprod Genet 34: 109-116, 2017.

14. Liu X, Xu Y, Sun J, Zhang Z, Wang J, Ding C, Zheng SL, Xu J and Zhou C: Preimplantation genetic haplotyping for six Chinese pedigrees with thalassemia using a single nucleotide polymorphism microarray. Prenat Diagn 37: 460-468, 2017.

15. Giménez C, Sarasa J, Arjona C, Vilamajó E, Martínez-Pasarell O, Wheeler K, Valls G, Garcia-Guixé E and Wells D: Karyomapping allows preimplantation genetic diagnosis of a de-novo deletion undetectable using conventional PGD technology. Reprod Biomed Online 31: 770-775, 2015.

16. Handyside AH, Harton GL, Mariani B, Thornhill AR, Affara N, Shaw MA and Griffin DK: Karyomapping: A universal method for genome wide analysis of genetic disease based on mapping crossovers between parental haplotypes. J Med Genet 47: 651-658, 2010.

17. Natesan SA, Bladon AJ, Coskun S, Qubbaj W, Prates R, Munne S, Coonen E, Dreesen JC, Stevens SJ, Paulussen AD, et al: Genome-wide karyomapping accurately identifies the inheritance of single-gene defects in human pre-implantation embryos in vitro. Genet Med 16: 838-845, 2014.

18. Ren Y, Zhi X, Zhu X, Huang J, Lian Y, Li R, Jin H, Zhang Y, Zhang W, Nie Y, et al: Clinical applications of MARSALA for preimplantation genetic diagnosis of spinal muscular atrophy. J Genet Genomics 43: 541-547, 2016.

19. Martín J, Cervero A, Mir P, Martinez-Conejero JA, Pellicer A and Simón C: The impact of next-generation sequencing technology on preimplantation genetic diagnosis and screening. Fertil Steril 99: 1054-1061.e3, 2013.

20. Yan L, Huang L, Xu L, Huang J, Ma F, Zhu X, Tang Y, Liu M, Lian Y, Liu P, et al: Live births after simultaneous avoidance of monogenic diseases and chromosome abnormality by next-generation sequencing with linkage analyses. Proc Natl Acad Sci USA 112: 15964-15969, 2015.

21. Chen L, Diao Z, Xu Z, Zhou J, Wang W, Li J, Yan G and Sun H: The clinical application of preimplantation genetic diagnosis for the patient affected by congenital contractural arachnodactyly and spinal and bulbar muscular atrophy. J Assist Reprod Genet 33: 1459-1466, 2016.

22. Gardner DK, Lane M, Stevens J, Schlenker T and Schoolcraft WB: Blastocyst score affects implantation and pregnancy outcome: Towards a single blastocyst transfer. Fertil Steril 73: 1155-1158, 2000.

23. Dreesen JC, Jacobs LJ, Bras M, Herbergs J, Dumoulin JC, Geraedts JP, Evers JL and Smeets HJ: Multiplex PCR of polymorphic markers flanking the CFTR gene; a general approach for preimplantation genetic diagnosis of cystic fibrosis. Mol Hum Reprod 6: 391-396, 2000.

24. Ou J, Wang W, Feng T, Liao L, Meng Q, Zou Q, Ding J, Zheng A, Duan C, Li P, et al: Identification of small segmental translocations in patients with repeat implantation failure and recurrent miscarriage using next generation sequencing after in vitro fertilization/intracytoplasmic sperm injection. Mol Cytogenet 8: 105,2015

25. Goyal M, Bijarnia-Mahay S, Kingsmore S, Farrow E, Saunders C, Saxena R and Verma IC: Molecular diagnosis of infantile neuro axonal dystrophy by next generation sequencing. Indian $\mathrm{J}$ Pediatr 82: 474-477, 2015.

26. Thornhill AR, Handyside AH, Ottolini C, Natesan SA, Taylor J, Sage K, Harton G, Cliffe K, Affara N, Konstantinidis M, et al: Karyomapping-a comprehensive means of simultaneous monogenic and cytogenetic PGD: Comparison with standard approaches in real time for Marfan syndrome. J Assist Reprod Genet 32: 347-356, 2015.

27. Liehr T: Cytogenetic contribution to uniparental disomy (UPD). Mol Cytogenet 3: 8, 2010.

28. Solomons J, Ridgway O, Hardy C, Kurian MA, Jayawant S, Hughes S, Pretorius P and Németh AH: Infantile neuroaxonal dystrophy caused by uniparental disomy. Dev Med Child Neurol 56: 386-389, 2014.

29. Goldman KN, Nazem T, Berkeley A, Palter S and Grifo JA: Preimplantation genetic diagnosis (PGD) for monogenic disorders: The value of concurrent aneuploidy screening. J Genet Couns 25: 1327-1337, 2016.

30. Tan Y, Yin X, Zhang S, Jiang H, Tan K, Li J, Xiong B, Gong F, Zhang C, Pan X, et al: Clinical outcome of preimplantation genetic diagnosis and screening using next generation sequencing. Gigascience 3: 30, 2014. 
31. Wells D, Kaur K, Grifo J, Anderson S, Taylor J, Fragouli E and Munne S: A novel embryo screening technique provides new insights into embryo biology and yields the first pregnancies following genome sequencing. Hum Reprod 28: i26, 2013.

32. Lledó B, Morales R, Ortiz JA, Blanca H, Ten J, Llácer J and Bernabeu R: Implantation potential of mosaic embryos. Syst Biol Reprod Med 63: 206-208, 2017.

33. Greco E, Minasi MG and Fiorentino F: Healthy babies after intrauterine transfer of mosaic aneuploid blastocysts. N Engl J Med 373: 2089-2090, 2015.

34. Besser AG and Mounts EL: Counselling considerations for chromosomal mosaicism detected by preimplantation genetic screening. Reprod Biomed Online 34 369-374, 2017.
35. Fiorentino F, Bono S, Biricik A, Nuccitelli A, Cotroneo E, Cottone G, Kokocinski F Michel CE, Minasi MG and Greco E: Application of next-generation sequencing technology for comprehensive aneuploidy screening of blastocysts in clinical preimplantation genetic screening cycles. Hum Reprod 29: 2802-2813, 2014

36. Handyside AH: 24-chromosome copy number analysis: A comparison of available echnologies. Fertil Steril 100: 595-602, 2013.

This work is licensed under a Creative Commons Attribution-NonCommercial-NoDerivatives 4.0 International (CC BY-NC-ND 4.0) License. 